Fall 2011

\title{
Le Zarathoustra de Nietzsche et le style parodique. A propos de l'hyperanthropos de Lucien et du surhomme de Nietzsche
}

Babette Babich

Fordham University, babich@fordham.edu

Follow this and additional works at: https://fordham.bepress.com/phil_babich

Part of the Ancient History, Greek and Roman through Late Antiquity Commons, Classical Literature and Philology Commons, Continental Philosophy Commons, German Language and $\underline{\text { Literature Commons, History of Philosophy Commons, and the Scandinavian Studies Commons }}$

\section{Recommended Citation}

Babich, Babette, "Le Zarathoustra de Nietzsche et le style parodique. A propos de l'hyperanthropos de Lucien et du surhomme de Nietzsche" (2011). Articles and Chapters in Academic Book Collections. 52.

https://fordham.bepress.com/phil_babich/52 


\title{
LE ZARATHOUSTRA DE NIETZSCHE ET LE STYLE PARODIQUE À PROPOS DE L'HYPERANTHROPOS DE LUCIEN ET DU SURHOMME DE NIETZSCHE
}

\author{
par
}

BABETTE BABICH

\section{Le Zarathoustra de Nietzsche, l'Empédocle de Nietzsche}

J'entreprends ici de lire Ainsi parlait Zarathoustra en résonance avec le Katharmoi, ou Purifications, un texte ésotérique d'Empédocle, à travers Lucien de Samosate, satiriste du II siècle, dont Nietzsche admirait le style exemplaire de la "satyra menippea ${ }^{11}$. Dans plusieurs de ses dialogues, Lucien met Ménippe au premier plan, comme Platon le fit pour Socrate, de sorte que l'on peut voir en lui un double de Lucien (et, comme avec Platon et Socrate, les chercheurs s'opposent dans leur estimation de Lucien visà-vis Ménippe) $)^{2}$. Le style parodique de Lucien atteint d'ailleurs son apogée lorsque Ménippe prend la parole et l'on peut avancer que cette peinture de Ménippe a fondé la réputation de Lucien comme celui qui dit la vérité en mentant ${ }^{3}$.

Bien qu'universellement connu et lu dans le passé, l'œuvre et le nom de Lucien sont devenus de plus en plus étrangers aux chercheurs, surtout en philosophie. Cette méconnaissance actuelle vaut pour l'ensemble du monde philosophique. Adoptant le point de vue de la philosophie analytique, Annette Baier observe à propos de David Hume que, "bien que Lucien eût été largement lu à l'époque de Hume, les passerelles entre les lecteurs de Hume et ceux de

1. Dans ce contexte de la philologie ancienne, Nietzsche remarque que la satire ménipéenne a été forgée ou "inventée" par les cyniques. Sur ce point chez Ménippe de Gadara, voir Diogène Laërte (1965 : VI, 46). Pour l'admiration de Nietzsche, voir Le Crépuscule des idoles, "Ce que je dois aux Anciens " $\$ 2$.

2. Voir également Clay (1992).

3. Remarquons que Lucien est décrit par Eupanius ( $V^{e}$ siècle) comme un menteur " sincère ", qui recherche ou vise la vérité. Voir aussi les récentes contributions à Ebner et al. (2001), particulièrement celle de Nesselrath, "Lukian und die antike Philosophie"(135-152). On peut également se reporter, plus largement, à Branham (1989) ainsi qu'aux contributions à Bartley (2009).

Diogène $\mathrm{n}^{\circ} 232$, octobre 2010. 
Lucien semblent aujourd'hui quasiment inexistantes $»^{4}$. Bien que Nietzsche, spécialiste de lettres classiques et philologue, ait toujours cité Lucien, depuis ses premiers travaux (La naissance de la tragédie) jusqu'à la fin de sa vie productive ("Ce que je dois aux anciens " du Crépuscule des idoles - titre lui-même assez lucianique), rares sont ceux qui lisent Nietzsche et Lucien.

Comme Baier le remarque très justement, Lucien est loin d'être un lieu commun en philosophie, ce qui mérite d'être souligné car il fut un temps où il comptait parmi ses lecteurs un grand nombre d'auteurs européens très différents les uns des autres : Érasme (qui écrit en latin) ${ }^{5}$, Goethe et Schiller pour l'Allemagne ; Voltaire ${ }^{6}$, Rabelais, Diderot, ainsi que de Bergerac et Montaigne, et évidemment Fontenelle avec ses Nouveaux Dialogues des morts (1683) comme Nietzsche le cite dans son Le Voyageur et son Ombre §214 (titre encore très lucianique) - pour la France ; Thomas More, mais aussi Shakespeare, Milton, Swift, Sterne et Joyce pour le Royaume-Uni et l'Irlande. Lucien a également influé sur des philosophes comme Hume, Marx, Hegel (pour qui il est précisément le «Grec Syrien " à ne pas admirer) et, bien sûr, Nietzsche.

Lucien a incarné beaucoup, voire trop de choses pour la plupart de ses lecteurs, ce qui signifie également qu'à l'instar de Nietzsche il est difficile à classer. Dans son essai consacré à l'influence de Lucien sur l'Utopie de Thomas More, Carlo Ginzburg (2005 : 31, cf. 30 sq.) a tenté d'expliquer les particularités et les dissonances de son influence. Il observe qu'« à partir de 1530, Lucien fut pour beaucoup, dans toute l'Europe (entre autres pour Calvin), synonyme de "mécréant, athée". " En réalité, le statut d'auteur parodique et la notion même de satire (ménippéenne ou non) suffisait à se faire taxer d'incroyance, jugement qui participe à la tendance anachronique ou présentiste ${ }^{7}$ d'aborder, à travers le filtre du protes-

\footnotetext{
4. Voir Baier (2008 : 103). L’intérêt de Baier pour Lucien, comme elle nous le dit, est né d'une demande du chercheur italien Emilio Mazza (110) à propos du titre exact du dialogue que lisait Hume sur son lit de mort. Adam Smith, la source habituellement citée à ce sujet, est pour Baier trop peu précis, lorsqu'il nous dit que "lorsqu'il a rendu visite à Hume le 8 août, celui-ci lisait les Dialogues sur la mort de Lucien» (p. 100). Le dialogue s'avère être le Kataplous de Lucien, qui est également, pour rendre justice à Smith, un "dialogue sur la mort", tous les personnages étant associés à la mort.

5 . Il est sur ce point significatif que Lucien ait été traduit en latin dès 1400 et aussi par Erasme en 1506. Pour ce qui est de Lucien en France, avec une nette allusion à Nietzsche, voir la publication en ligne de Philippe Renault (2004).

6. Voir « Conversation de Lucien, Érasme, et Rabelais dans les Champs Élysées " (1765).

7. Il s'agit d'une histoire « whig », ou « whiggish » selon Butterfield (1951).
} 
tantisme, l'histoire de la philosophie de Xénophane et Anaxagore aux Lumières, en passant par Platon, Socrate et plus encore Aristote - à savoir, tous ceux habituellement accusés (à tort ou à raison) de scepticisme envers les dieux grecs8. Étant donné l'« athéisme " avoué de Lucien vis-à-vis des dieux supposés païens comme du Dieu judéo-chrétien, Ginzburg (ibid.) reformule sa question et se demande: "que signifiait Lucien quelques décennies auparavant pour Érasme et pour More ${ }^{9}$ ?"

Je vais défendre ici l'idée selon laquelle un parallèle ironique peut être tracé entre l'hyperanthropos satirique de Lucien et l'idéal sérieux ou élevé du surhomme nietzschéen, idéal dont notre approche est trop souvent privée d'ironie ${ }^{10}$. Je trouve cette ironie éclairante puisque c'est justement la particularité de Lucien, comme celle de Nietzsche, de mélanger les registres. De la même manière, Ginzburg souligne qu'Érasme loue le talent de Lucien, qui mêle « le sérieux et le frivole, et le frivole avec les choses sérieuses (sic seria nugis, nugas seriis miscet) ${ }^{11}$." L'hyperanthropos de Lucien est un concept hautement métonymique dont le sens originel contient son en-deçà et son au-delà : il signifie à la fois sur-humain, au-dessus du simplement humain, et surhomme apparent, avec tous les attributs de l'homme supérieur, - c'est « par leurs fruits que vous les reconnaîtrez " devient "c'est par leur cuisine que vous les reconnaîtrez ». Dans le texte de Lucien, nous suivons cet homme supérieur (ici le tyran Mégapenthès), supérieur comme on le qualifie sur terre, c'est-à-dire dans le monde du dessus, et l'on assiste à sa "translation ", c'est-à-dire à son passage dans l'Hadès ou les Enfers. Il s'agit de son "voyage vers les abîmes ", parmi les ombres, comme les Grecs imaginent la vie après la mort : un domaine sombre et obscur, qui ne diffère guère des profondeurs sans soleil du She'ol hébraïque.

Comme je l'ai déjà rappelé, ce n'est pas une simple coïncidence si Nietzsche a étudié la philologie classique. Il était l'un des meilleurs spécialistes de cette discipline à son époque. Je soutiens et j'ai soutenu ailleurs que la philologie est restée sa vocation tout au

8. Remarquons ici que ne pas croire aux dieux ne revient pas à dire qu'il n'y a pas de dieux, mais à refuser le polythéisme.

9. Le livre de Christine Lauvergnat-Gagnière (1988), est également pertinent dans ce contexte.

10. Voir, par exemple, l'entrée Übermensch dans le Handwörterbuch der Philosophie de Rudolf Eisler (1904), qui s'ouvre par: "Übermensch ist eigentlich nichts anderes als die Idee des vollkommeneren, des vollkommensten Menschen, sowohl als Gattung wie auch als Individualität (Genie) gedacht. "

11. Ginzburg (2005 : 33). La version anglaise comprend la citation du latin, ce qui n'est pas le cas dans l'édition italienne originale ni dans la traduction française. 
long de son existence ${ }^{12}$. Non seulement Nietzsche connaissait, comme il se doit, Lucien ${ }^{13}$ - contrairement à de nombreux philologues actuels -, mais il existe des parallèles évidents entre certaines tournures d'esprit typiques de Nietzsche et certaines des idées favorites de Lucien, à commencer par un attrait commun pour la démystification ${ }^{14}$. On peut ainsi comparer les fameuses remarques de Nietzsche sur la vérité et le mensonge avec l'Alethe Diegemata ou Histoire véritable de Lucien qui comprend le paradoxe crétois "Je ne dis pas un seul mot de vrai " (Lucien de Samosate 1896 : 431 ; cf. Lucien de Samosate 1866).

Lucien affirme ici (1896:431) que ce qui rend son exposé différent est qu'il propose " un mensonge beaucoup plus raisonnable que ceux des autres ». Comme Nietzsche le fera plus tard, Lucien annonce ouvertement sa prévarication comme une variante manifeste et donc supérieure aux mensonges traditionnels exposés par les autres historiens dans son Histoire véritable:

J'en userai du moins avec plus de probité que les autres : et quand mon ouvrage ne contiendrait d'autre vérité que l'aveu que je fais qu'il ne contient que des mensonges, j'éviterai, du moins, le reproche que je faisais tout à l'heure aux autres (ibid.).

Prenant le contre-pied de Platon, et étant donné la référence de Lucien aux philosophes (" mentir est un usage consacré par ceux-là mêmes qui se donnent pour philosophes ", ibid.), il serait difficile de ne pas prendre en compte l'aspect épistémologique de son avertissement : “ je vais donc raconter des faits que je n'ai pas vus, des aventures qui ne me sont pas arrivées et que je ne tiens de personne ; j'y ajoute des choses qui n'existent nullement, et qui ne peuvent pas être : il faut donc que les lecteurs n'en croient absolument rien. ")

Le ton de Lucien n'est bien sûr pas celui de Nietzsche, mais l'enjeu épistémologique est identique, si l'on se réfère à la célèbre déclaration de Nietzsche contre la vérité comme valeur suprême au

12. Voir, pour une analyse, Babich (2005). Voir plus particulièrement, pour des références, p. 48-49.

13. En plus des propres références de Nietzsche à Lucien, voir l'ami de Nietzsche, Erwin Rohde (1869). Nietzsche comme Rohde se réfèrent à Lucien dans leur correspondance. Bien que Lucien n'en soit pas le sujet principal, voir, pour une analyse de Rohde et Nietzsche, Cardew (2004). Il existe chez Nietzsche un grand nombre de références à Lucien (certaines déjà données, d'autres à venir dans les notes suivantes), mais Friedrich Kainz nous rappelle qu'en plus des origines de l'Übermensch, que pour sa part Kainz fait remonter, pour ce qui est de la forme la plus ancienne, à Herder et Goethe, Lucien est également la source du langage " alcyonien ». Voir Kainz (1974 : 340). Pour un amusant exemple, voir Rauer (2005).

14. Sur cette notion de démystification, voir Wallas (1994 : chapitre dix). 
début de ses réflexions dans Par-delà le Bien et le Mal : «Étant admis que nous voulons la vérité, pourquoi pas plutôt la nonvérité ? ${ }^{15} \mathrm{Si}$ ce présupposé initial de vouloir le vrai peut sembler adventice (une inquiétude pour certains puisque sa préface commence par: "À supposer que la vérité soit femme, n'a-t-on pas lieu de soupçonner que tous les philosophes, pour autant qu'ils fussent dogmatiques, n'entendaient pas grand-chose aux femmes [...]? " [PBM §1]), Nietzsche, de manière systématique - et il faut remarquer qu'il est perçu non seulement comme un philosophe sans système, mais comme un de ceux qui s'opposent explicitement à ce qu'il appelle la "volonté " de système - applique les mêmes remarques aux logiciens, aux mathématiciens, et, après cela, aux physiciens ou cosmologistes. Du fait que "le déterminé a plus de valeur que l'indéterminé, ou que l'apparence vaut moins que la "vérité" ", il affirme que leurs évaluations, ou estimations, peuvent être vues comme «des jugements superficiels (doch nur VordergrundsSchätzungen], une sorte particulière de niaiserie " (ibid.) La transition vers l'épistémologie évolutionniste ayant déjà commencé au XIXe siècle, l'argument de Nietzsche selon lequel la pure conjoncture que "l'homme ne pourrait pas vivre sans se rallier aux fictions de la logique, sans rapporter la réalité au monde purement imaginaire de l'absolu et de l'identique, sans fausser continuellement le monde en y introduisant le nombre " (PBM $§ 4$ ), ne modifie en rien la nature de ces falsifications. Au-delà du formel et de l'idéal, Nietzsche soutient qu'il n'est pas le seul (nous pourrions mentionner Poincaré ou Mach) ${ }^{16}$ à supposer que "la physique n'est, elle aussi, qu'une interprétation du monde, une adaptation du monde " et non une " explication du monde" (PBM §14). En ce qui concerne sa philosophie de la science, j'ai défendu ailleurs l'idée qu'à cet égard la cohérence de Nietzsche est d'une rigueur philosophique vraiment frappante. Il affirme, pas si loin de Xénophon ou d'Héraclite et Épicure, mais surtout de David Hume : «c'est nous seuls qui avons forgé les causes, la succession, la réciprocité, la relativité, la nécessité, le nombre, la loi, la liberté, le mobile, la finalité " (PBM §21). Nietzsche vise Kant en disant que lorsque "nous incorporons ce monde de signes aux choses "en soi", nous retombons dans l'erreur où nous sommes toujours tombés : la mythologie" (ibid.). Il se met et nous met continuellement en garde contre cette erreur et c'est dans ce contexte qu'il insiste sur ce point, en s'adressant « au vieux

15. Nietzsche (1971, cité ici, non par pages, mais par numéro de section, comme PBM : ici, PBM 1). [Traduction modifiée].

16. Je suis souvent revenue sur le contexte de la science du XIXe siècle selon les termes de la philosophie de la science de Nietzsche. Pour un récent exemple plus général, voir Babich (2010), et plus particulièrement la section " Nietzsche and the Sciences" (353 sq.). 
philologue ». Il s'oppose à la projection ultra-scientifique d'une conformité de la nature à des lois et nous taquine avec son "vous, physiciens, parlez avec tant d'orgueil » (PBM §22). Pour Nietzsche, une telle "conformité » à des lois résulte d'une interprétation : «Elles n'ont aucun contenu réel, elles ne correspondent à aucun "texte" ; ce sont bien plutôt des arrangements et des falsifications naïvement humanitaires " (ibid.). Nietzsche concède ainsi avec joie que n'importe qui pourrait lui rétorquer : "ceci aussi n'est qu'une interprétation ", ce à quoi il répondrait : " eh bien, tant mieux » (ibid.) $)^{17}$.

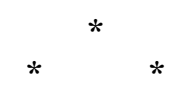

Je me concentrerai maintenant sur un dialogue particulier de Lucien, le Kataplous (diversement traduit par Le passage de la

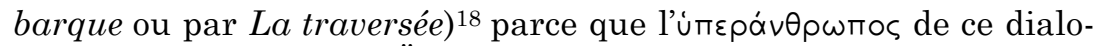
gue est la source de l'Übermensch de Nietzsche, le surhumain ${ }^{19}$. Au-delà de cette attribution très conventionnelle et de sa réception, je soutiens que le contexte de La traversée de Lucien, y compris son sous-titre thématique, ou le Tyran - puisqu'il s'agit d'un dialogue en forme de vanitas sur le destin des âmes (en particulier celui du tyran dans le monde des Enfers, qui s'oppose au monde d'en-haut) - illustre l'éternel ou ultime contexte du " doit » de l'enseignement de Zarathoustra, pour qui l'humain doit être dépassé.

On pourrait souligner également l'intérêt de Nietzsche, via Lucien, pour les saveurs de la cuisine ou pour la cuisine elle-même (et par extension métonymique pour les entrailles et les aspects physiologiques de la nutrition, telle la digestion) ${ }^{20}$. Parallèlement aux analyses de Nietzsche sur la nourriture, la lecture de Lucien révélerait l'importance de la formation philologique de Nietzsche. Ainsi, dans le quatrième livre de Zarathoustra, la cène du " souper "

17. Cette approche de la notion de vérité traverse toute l'œuvre de Nietzsche.

18. Ici, hormis la traduction traditionnelle, je suis la nouvelle traduction de Philippe Renault (Lucien 2005).

19. Il s'agit de loin de la plus classique attribution, mais j'y reviendrai plus loin. Pour l'heure, je tiens à remercier Eli Friedländer et Horst Hutter pour avoir remarqué la plus grande complexité de cette question déjà complexe. Ainsi, par exemple, Claude Pavur (1998) lit l'Übermensch dans le contexte humaniste de la Renaissance. On peut également penser au surhomme, dans l'esprit de Montanus, comme Attis ou comme le Christ, avec un parallèle évident dans le berger du Zarathoustra.

20. Derrida est sur ce point une source inestimable d'inspiration pour de nombreuses réflexions, là où il fait appel au Zarathoustra de Nietzsche (et n'oublions pas Brillat-Savarin à l'arrière-plan) dans son interview avec Jean-Luc Nancy (Derrida 1989). 
inclut les mets les plus raffinés. Cette occurrence sert à montrer les desiderata de Zarathoustra: de l'agneau aromatisé à la sauge et servi avec du vin (en opposition avec le repas végétarien de grain moulu et d'eau), le tout à consommer dans une atmosphère de joie et de plaisir - et n'oublions pas que la figure historico-mythique de Zarathoustra serait née en riant. Cette mention de la bonne humeur bat en brèche l'idée de supériorité morale et jette un doute sur les vertus morales de ces plaisirs de la table (et manger de la viande reste toujours moralement délicat). Ainsi, cette section parodique du quatrième livre d'Ainsi parlait Zarathoustra ne pose pas seulement le décor de celle intitulée (et comment l'intituler autrement?) "De l'homme supérieur", mais contient aussi une allusion claire à l'équation (très lucianique) qui met à égalité les cuisiniers et les rois, (" Car chez Zarathoustra même le roi peut être cuisinier " [Z IV, La Cène $]$ ). Cette conversion démocratique fait aussi écho au thème du Kataplous de Lucien, qui contient non seulement une analyse de la cuisine et des odeurs qui en émanent, mais une théâtralisation du renversement des conditions des cordonniers (comme Micyllus) et des tyrans (comme Mégapenthes).

Si les vocations en question (celles des cordonniers et des dirigeants) sont des allusions à la République de Platon, les résonances chrétiennes s'avèrent également importantes chez Lucien ${ }^{21}$. En outre, ces mêmes allusions nous invitent à voir en Zarathoustra une figure explicitement empédocléenne. Le début du Katharmoi propose une liste des vies ou des rôles que chacun doit endosser (prophète, poète, soigneur) afin de mourir libre et d'aller en terre émancipé de sa condition de mortel. Ainsi parlait Zarathoustra raconte la descente aux enfers du personnage éponyme par celui-là même qui meurt - mais le fait sur un mode philosophique, dans la mesure où philosopher consiste à apprendre à mourir.

De la même manière qu'Empédocle "parle " à ses Agrigentiens, et leur apprend la doctrine de l'éternel renouveau, Zarathoustra est celui qui enseigne l'éternel retour et bénit la vie, transmettant la leçon du surhomme et du dépassement de soi, par-delà la ritournelle des naissances et des renaissances, jusqu'à une éternité immuable, amor fati, da capo.

En proposant de lire Ainsi parlait Zarathoustra entre le Katharmoi d'Empédocle et la Traversée de Lucien, je rejoins inévitablement les nombreux chercheurs qui défendent l'idée que le Zarathoustra de Nietzsche est calqué sur quelque chose ${ }^{22}$ - que ce soit la Bible ou la République de Platon, voire le Ring de Wagner, comme

21. Voir, pour un exemple d'une telle analyse théologique du renversement, Ronald F. Hock (1987).

22. C'est en ce sens général que l'on parle habituellement de parodie. Pour plus de références en anglais, voir notamment Wolfe (1964). 
on le lit chez Bertram et d'autres. Même si de tels parallèles ont leurs limites, j'affirmerai, dans la veine de Ginzburg, qu'ils sont nécessaires pour comprendre la pensée de Nietzsche sur les thèmes développés dans Zarathoustra, c'est-à-dire le surhumain, la volonté de puissance, et l'éternel retour du même.

Nous avons besoin de Lucien non seulement pour son art de la satire ou de la parodie mais également pour sa peinture d'Empédocle, entre autres. Nous avons besoin de Lucien tout simplement parce que son œuvre embrasse, par la lettre et l'esprit, l'antiquité gréco-romaine dans sa totalité - ses dieux et ses philosophes - ainsi que, et ce point est capital, le nouveau Dieu du Nouveau Testament, car ce n'est qu'avec cette nouvelle foi que la vieille croyance germanique (dont Nietzsche parle en disant «tous les dieux doivent mourir» KSA 7, 5 [115] $)^{23}$ est finalement, inéluctablement, accomplie - consummatum est, tout est achevé.

Des Sectes (philosophiques) à l'encan de Lucien à la "ligne d'or " de Zarathoustra

Avant d'aborder l'Empédocle de Nietzsche, il faut noter que Le pêcheur ou les ressuscités de Lucien a influé sur Ainsi parlait Zarathoustra de Nietzsche. Le texte de Lucien raconte l'histoire de la résurrection de philosophes morts depuis des siècles ${ }^{24}$, des présocratiques à Socrate lui-même, ainsi que Platon, Aristote et Diogène le cynique. Tous se voient libérés du monde souterrain l'espace d'une journée afin de retourner dans le " monde supérieur » (c'està-dire à la vie sur terre) pour se venger des moqueries de Lucien dans son dialogue précédent, Les sectes [philosophiques] à l'encan. Ce dialogue haut en couleurs s'ouvre par l'expression de rage d'un Socrate temporairement ressuscité qui s'écrie : " que l'infâme [Lucien] porte la peine pour ses calomnies 25 !"

Cette satire est un exercice agonistique, le agon en question étant indispensable pour éclaircir ce qui sinon resterait une référence sibylline à la "canne à pêche dorée" dans L'offrande $d u$ miel, sans parler de la métaphore de la pêche et des " hameçons", récurrente chez Nietzsche. Ce dernier rappelle l'importance de la métaphore de la pêche (d'habitude renvoyant au Christ " pêcheur d'hommes ») dans Ecce Homo : "Depuis lors, tous mes écrits sont

23. Voir la discussion d'Isabelle Wienand (2006).

24. Comme nous le soulignions plus haut à propos des remarques de Baier sur le dialogue que Hume lisait sur son lit de mort et l'identification d'Adam Smith, les dialogues avec les morts constituent le fond de commerce de Lucien. La Traversée, ainsi que Les ressuscités, peuvent donc être considérés comme autant de " dialogues avec les morts ".

25. Lucien de Samosate (1896:238) ; cf. Lucian (1913-67 : III, 3). Talbot, traduit quant à lui : " que le calomniateur soit puni comme il le mérite ». 
des hameçons : peut-être suis-je aussi doué que quiconque pour la pêche à la ligne ? " (EH, Par-delà Bien et Mal, §1). Ici, la ligne dorée apparaît dans la première section du quatrième livre, volontairement non publié, d'Ainsi parlait Zarathoustra, suivi du Cri de détresse et du Dialogue avec les rois.

Le pêcheur de Lucien nous offre donc ce que l'on peut voir comme un "essai d'autocritique", parodiant le destin de ses propres parodies face aux philosophies qu'il met pour ainsi dire " aux enchères ". La "ligne dorée " [goldene Angelruthe] sert d'appât pour tester le sang-froid des philosophes, mesurer leurs mérites, évaluer leur honnêteté (souvent relative), les faire comparaître devant la Vérité et la Philosophie personnifiées, en compagnie de Diogène de Sinope, le Cynique par excellence dont la simplicité du langage n'a d'équivalent que celle de sa vie. Dans le contexte de Zarathoustra, l'image du test auquel sont soumis les prétendants à la philosophie chez Lucien est intéressante car c'est la Philosophie elle-même qui donne le détail de l'épreuve à Vérité [Aletheia] et Franchise [Parrhesia] :

Éprouvons-les, Parrhésiade, comme on dit que les aigles éprouvent leurs petits aux rayons du soleil. Cependant, ce n'est pas en leur faisant fixer la lumière qu'il faut éprouver les philosophes, mais en leur présentant de l'or, de la gloire, des voluptés. Celui que tu verras n'y point arrêter sa vue et qui se montrera insensible à leur aspect, c'est celui-là qu'il faudra couronner. (Lucien 1896 : 262 ; cf. Lucien 1913-67 : $69)$.

Le problème avec ceux qui se nomment philosophes est précisément qu'en dépit des "maximes, qu'ils ont sans cesse à la bouche, sur le mépris qu'on doit avoir pour les richesses et la vaine gloire, sur ce qu'il faut n'estimer utile que ce qui est honnête, s'abstenir de la colère, n'avoir aucun respect pour les grands, et leur parler comme à ses égaux", ils " n'enseignent que pour un salaire ». Bien plus encore :

ils s'extasient à la vue des gens riches, sont avides d'argent, d'ailleurs plus colères que les petits chiens, plus lâches que les lièvres, plus flatteurs que les singes, plus lascifs que les ânes, plus voleurs que les chats, et plus querelleurs que les coqs. Ils méritent bien qu'on rie à leurs dépens, lorsqu'on les voit courir avec empressement vers tout ce qu'ils défendent, se porter en foule à la porte des riches, rechercher les festins splendides, s'y livrer à la flatterie la plus intrépide, se remplir l'estomac plus que l'honnêteté ne le permet, faire éclater leur mauvaise humeur, philosopher au milieu des pots, de la manière la plus ridicule et la plus déshonnête, et finir par ne pouvoir plus contenir l'excès du vin qu'ils ont bu. (Lucien 1896 : 255-256).

Pour séparer les faux prétendants à la philosophie des vrais, nous lisons l'échange dialogué de Lucien sur les exigences du test en lui-même : 
Parrhésiade. - ... si la Prêtresse veut me prêter, pour un instant, la ligne et l'hameçon que le Pêcheur du Pirée a consacrés à Minerve.

La Prêtresse. - Les voilà avec le manche de Roseau, afin que tu aies tout. (Lucien $1896: 262$ ).

Appâté par "des figues et un petit morceau d'or ", le premier " poisson " attrapé sous les yeux du Diogène ressuscité est en fait " un chien marin " (ibid.) ${ }^{26}$. Le poisson-chien ou cynique est suivi d'un platonicien, d'un imposteur aristotélicien, etc., " poissons " qui au cours du dialogue sont durement condamnés pour leurs faiblesses par les mêmes philosophes qui au début du dialogue étaient revenus à la vie pour persécuter Lucien, mais qui finissent par admettre que ses dénonciations s'appliquaient très bien aux soidisant "philosophes" de son temps. La tentation est grande d'affirmer que notre époque n'est guère différente.

Quand le Zarathoustra de Nietzsche parle du " meilleur appât ", il veut dire " comme il en faut aux chasseurs et aux pêcheurs " et lorsqu'il se réfère à " une mer pleine de multicolores poissons et crustacés ", il parle dans les mêmes termes choisis d'une richesse " de laquelle même des dieux voudraient avoir envie pour s'y faire pêcheurs et y jeter filet - si riche est le monde en merveilleux, grand et petit!" (Zarathoustra IV, "L'offrande du miel»). Cette manière de s'exprimer est lucianique et mélange la langue de la littérature classique et de ses dieux avec le langage du Nouveau Testament, auxquels s'ajoute la rêverie parodique de Nietzsche lancé à la recherche de ceux à qui il pourrait parler - ses lecteurs, ses êtres humains :

Singulièrement, le monde humain, l'humaine mer - en laquelle à présent je jette ma ligne d'or et dis : Ouvre-toi, abîme humain!

Ouvre-toi et me lance tes poissons et tes scintillants crustacés ! De mon meilleur appât je m'appâte aujourd'hui les plus merveilleux poissons-hommes!

...Jusqu'à ce que, mordant à la pointe de mon secret hameçon, il leur faille s'élever jusqu'à ma cime, les plus multicolores goujons d'abîme, jusques au plus cruel de tous les pêcheurs d'hommes (ibid.)

\section{La mort de Zarathoustra et les ébauches de Nietzsche} pour la Mort d'Empédocle

Dans une partie intitulée « Les philosophes de l'époque tragique dévoilent, comme la tragédie, le monde " (KSA 7, 21 [16], 527 ; KGW III/4, 21 [16], 119), Nietzsche esquisse la relation entre Héraclite ("L'art au service de la volonté " [Kunst im Dienst des Willens ]), Empédocle ("Amour et haine en Grèce " [Liebe und Haß in Grie-

26. Salmo Cynicus : "Ah dieux, quelles dents !... Eh, eh ! Il n’y a plus rien à l'hameçon. Le drôle a avalé la figue et l'or. » 
chenland]), et les autres présocratiques. Il qualifie Empédocle non seulement d'" idéal du Grec accompli » [ideal-vollkommene Grieche] (KSA 7, 3 [84], 83), mais aussi d'« homme tragique le plus pur» [reine tragische Mensch] (KSA 7, 5 [94], 118) et annonce le plan de son projet pour la "mort" d'Empédocle, qui se conclut par "Le grand Pan est mort " [Der große Pan ist tot] (5 [116], 125). Le parallèle avec Zarathoustra est flagrant.

Empédocle et Zarathoustra sont tous deux comparés au divin et simultanément plongés dans la mortalité. Empédocle accède à la divinité en mourant, élu ou " promu " (son refus de la royauté participe de cette accession) à travers même son statut d'exclu : « Je suis maintenant l'un de ceux-ci, un banni et un homme errant loin des dieux, car je mettais ma confiance dans la Haine insensée " (Empédocle $1934: 115)^{27}$.

David Allison, entre autres, rappelle qu'à l'origine Ainsi parlait Zarathoustra comprenait aussi la mort de Zarathoustra. Mais on peut peut-être aller plus loin, au-delà du figuré, et comprendre la référence lucianique au déclin de Zarathoustra dans le Prologue de Zarathoustra comme annonçant l'éventualité de la mort de Zarathoustra dans le premier livre du texte publié où Zarathoustra succombe à la morsure d'un serpent sous un figuier (un cliché métaphorique s'ajoutant à un autre) : "Ta route n'est que courte, dit tristement la vipère; mon poison tue" (Z I, De la morsure de la vipère).

De manière très explicite, traçant maintenant le parallèle avec Empédocle, Zarathoustra se dit à lui-même : "En vérité, de par cent âmes j'ai cheminé, et de par cent berceaux et cent gésines. Déjà j'ai dit bien des adieux, je connais les ultimes instants qui déchirent le cœur" (ibid.; cf. DK 31). Mais, " ainsi veut mon vouloir créateur, mon destin. $\mathrm{Ou}$, pour vous le dire plus loyalement, c'est ce destin précisément que - veut mon vouloir" (ibid.). De plus, l'enseignement d'Empédocle de la renaissance a des résonances dans le langage du " nuptial anneau des anneaux, - de l'anneau du retour" (Z III, Les Sept Sceaux ou Le Chant du Oui et de l'Amen).

Zarathoustra enseigne le sur-humain de même que la transition que constitue le surhumain et l'éternel retour du même. Il décrit l'homme comme étant " une corde tendue entre bête et surhumain - une corde sur un abîme ", incarnée par le funambule. Si la totalité du discours de Zarathoustra est un inévitable et involontaire commentaire sur la "performance" du danseur de corde et de sa propre chute, nous sommes confrontés à une dramatisation évidente de l'en-haut et de l'en-bas, c'est-à-dire: de l'ésotérique et de

27. Je développe ailleurs cette question de la royauté terrestre et de la résonance entre les utilisations que font Nietzsche et Hölderlin de l'affirmation que le temps des rois est révolu. 
l'exotérique 28 . C'est dans ce contexte, alors que le funambule évolue au-dessus de la foule et crée ainsi une tension, que Zarathoustra déclare qu'il est pour l'être humain " dangereux de passer, dangereux d'être en chemin, dangereux de se retourner, dangereux de trembler et de rester sur place ". Il enseigne du coup le surhumain dans un sermon où "le pont arc-en-ciel» de la vie est présenté comme un drame sur la vie et la mort se déroulant au-dessus de sa tête :

J'aime ceux qui ne savent vivre qu'en déclinant, car ils vont audessus et au-delà.

J'aime les grands contempteurs, car ils sont grands vénérateurs et vers l'autre rivage flèches de nostalgie.

J'aime ceux qui seulement au-delà des astres ne cherchent une raison de décliner et d'être hostiles, mais ceux qui à la Terre se sacrifient pour que la Terre un jour devienne celle du surhomme

J'aime celui dont l'âme se prodigue, qui ne veut gratitude et point ne rend, car toujours il prodigue et ne se veut garder (Z I : Prologue §4).

Cet enseignement devrait rappeler au lecteur le discours de l'ombre de Nietzsche dans le livre appendice d'Humain trop humain, Le voyageur et son ombre, dans lequel l'ombre tourne et s'évanouit. Nietzsche dit de l'être humain qu'il est un petit animal débordé qui, par chance, a eu aussi son heure ; la vie sur terre en tant que telle, éclipsée en un clignement d'yeux, un détail, "un instant, un accident, une exception sans suite [...] la terre même, comme tous les astres, un hiatus entre deux néants" [ein Zwischenfall, eine Ausnahme ohne Folge, [...] Erde selbst, wie jedes Gestirn, ein Hiatus zwischen zwei Nichtsen]29, " un événement sans plan, raison, volonté, conscience de soi, la pire sorte de nécessité, la stupide nécessité ..." [ein Ereigniß ohne Plan, Vernunft, Wille, Selbstbewußtsein, die schlimmste Art des Nothwendigen, die dumme Nothwendigkeit ...] (KSA 13, 16 [25], 488-489; cf. KSA 12, 10 [34], 473).

Dans la mesure où Zarathoustra enseigne ce que la philosophie enseigne - l'art de vivre -, il enseigne que le surhumain est " le sens de la Terre", c'est-à-dire que "l'homme est quelque chose qui se doit surmonter " (Z I : Prologue §3). Or l'art de vivre est aussi l'art d'apprendre à mourir, de la bonne manière, pour la bonne raison et « à la bonne heure " (Z I : De la libre mort).

28. Voir l'explication de Nietzsche sur "l'exotérique» versus «l'ésotérique ": "le point principal est que celui-ci regarde les choses de bas en haut, tandis que le penseur ésotérique les considère de haut en bas " (PDM $\S 30)$.

29. Nietzsche apprécie cette métaphore, qu'il utilise plus d'une fois. 
Da capo : le Surhumain de Nietzsche et l'hyperanthropos de Lucien

Il est communément admis que l'Übermensch nietzschéen correspond, de manière plus ou moins fortuite, au fantasme du III Reich hitlérien : il serait le sommet, en termes d'évolution, du développement humain (Habermas 2001). On a ainsi supposé que l'Übermensch était un être humain supérieur (ce qui veut aussi dire, avec Platon, Aristote, et même avec Alasdair MacIntyre, un guerrier supérieur, un soldat parfait), ou du moins de bonne race, ce par quoi on entend issu d'une famille assez aisée, et ayant bénéficié d'une " bonne " éducation, au sein d'un environnement favorable. L'Übermensch actuel apparaît comme la promesse de la science. À l'époque nazie, cet idéal scientifique était formulé en termes d'eugénisme, de génétique et de planification sociale. Aujourd'hui la science a franchi plus d'une de ces étapes, d'abord vers la génomique, puis vers la cybernétique - expliquant, dans notre société mue par le fantasme d'une ingénierie génétique et de ses technologies apparentées, l'enthousiasme persistant pour les cyborgs, considérés, dans leurs dernières incarnations, comme des posthumains, des transhumains. Si Nietzsche s'oppose à la conviction du darwinisme que les espèces actuelles représentent des "améliorations " ou des "développements " par rapport aux espèces du passé, il associe comme Darwin les humains et les singes. Autrement dit, quand le Zarathoustra de Nietzsche déclare : "Je vous enseigne le surhomme. L'homme est quelque chose qui doit se surmonter » (Z I : Prologue §3), il parle de dépasser l'humanité d'aujourd'hui avec son idéal de supériorité et de réalisation. Zarathoustra le rappelle : «Du ver de terre vous cheminâtes jusques à l'homme, et grandement encore avez en vous du ver de terre. Jadis vous fûtes singes et maintenant encore plus singe est l'homme que n'importe quel singe" (ibid.)

Lorsque nous entendons parler du surhomme, du surhumain, voire du transhumain, notre réaction est souvent de vouloir être les premiers; bien plus, nous avons la tentation de nous imaginer nous-mêmes déjà arrivés, d'être déjà des cyborgs, des transhumains, bref le couronnement de l'évolution : et comme ceci, des surhumains. Il est ainsi lourd de sens que les nietzschéens comme les antinietzschéens partagent la même définition du surhumain comme être "supérieur", sans pour autant s'entendre sur son sous-texte fasciste. La science ne cesse de nous rappeler que nous sommes une espèce supérieure, à commencer par rapport au singe. Nous admettons ou assumons aussi quotidiennement, au moins dans un contexte politique, notre propre supériorité culturelle/nationale/raciale sur les autres. Dans l'ensemble, la plupart d'entre nous n'ont pas besoin d'être convaincus que l'évolution marche en direction du surhumain (c'est nous). Et si nous n'avons 
pas encore accédé à ce statut supérieur par le biais de l'évolution naturelle, ou par défaut - étant donné la rapacité de destruction ou d'" utilisation " des ressources naturelles (par laquelle nous agissons comme si notre intention était d'être les derniers êtres à la surface de la terre, ce qui, il est vrai, peut être une façon de comprendre le "dernier homme " de Nietzsche) -, nous nous attendons à être transformés (ce serait une version de la singularité technologique) en "trans-(sur)-humains". En désaccord total avec à la fois les pro- et les antinietzschéens, Nietzsche lui-même, à mon sens, semble suggérer que surhumain, qua idéal trop humain, peut s'avérer être moins que ce que nous pensons. Pour comprendre ce point, nous devons faire un "pas en arrière ", pas pouvant entraîner un changement de perspective.

Tout spécialiste sait que l'Übermensch de Nietzsche est une création issue ou dérivée de Lucien. Tout spécialiste " sait " cela non pas parce que les spécialistes d'aujourd'hui lisent Lucien, mais parce que Walter Kaufmann nous l'a expliqué. Tout ce que j'ai pu lire sur le sujet cite Kaufmann (je le fais aussi), même si sa citation est très simple : «Kataplous, $16 »^{30}$. Dans ce qui précède, j’ai défen-

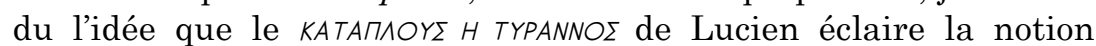
nietzschéenne de "surhumain". Le théoricien de la littérature Northrop Frye (1969 : 283) a étudié en détail ce type de comparaison, expliquant dans une section d'Anatomie de la critique que :

Lorsque la satire dépeint le "monde de l'au-delà ", elle le présente ironiquement comme l'antithétique de notre monde, lui attribuant des normes sociales entièrement opposées à celles que nous connaissons. C'est cette forme de satire qui est utilisée par Lucien dans ses dialogues, Kataplous et Charon, voyage dans l'au-delà où les grands de ce monde se conduisent d'une façon qui, pour être appropriée, ne nous semble pas moins totalement insolite; Rabelais utilise le même procédé que l'on retrouve également au Moyen Âge dans la danse macabre. Dans celle-ci, la très simple égalité de tous dans la mort est opposée aux complexes inégalités de la condition des vivants.

Il est à noter que, dans sa monographie sur le roman grec ancien et ses antécédents, Rohde, ami de Nietzsche, a pointé l'importance et l'étrange signification de la notion de "Voyager aux Enfers, pour l'amour de la connaissance philosophique " (1900: 261). C'est cette aventure souterraine qui apparaît à la fois chez Nietzsche (Zarathoustra) et chez Lucien, et c'est aussi l'instant de transfiguration du saut d'Empédocle dans le volcan.

30. Une note de Kaufmann (1974: 307 n. 1) clarifie le texte : «L'hyperanthropos se retrouve dans les écrits de Lucien au $\mathrm{II}^{\mathrm{e}}$ siècle après J.-C. et Nietzsche, en tant que philologue classique, a étudié Lucien et y fait de nombreuses références dans sa philologie ". Kaufmann ne propose pas de contexte, peut-être parce qu'il s'intéresse surtout à la création elle-même. 
L'opposition établie par Lucien dans le " Passage de la Barque " du Kataplous présente la vision superficielle d'un surhumain appartenant à la classe "supérieure", la classe des riches qui méprisent ceux qu'ils considèrent comme subalternes ou inférieurs sur

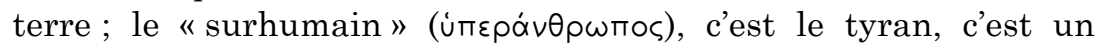
homme de pouvoir : "il me paraissait égal aux dieux. " Ces mêmes attributs politiques permettent à Micyllus, notre cordonnier, de rapporter que dans la vie, le tyran Mégapenthès était à ses yeux " au-dessus de la condition des hommes, et peu s'en fallait, à sa démarche altière, au respect et à la crainte qu'inspirait sa présence, que je ne le crusse le plus beau des mortels ; sa fortune semblait accroître sa taille d'une coudée royale" (Lucien $1896: 276$ ) (u்

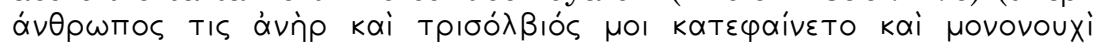

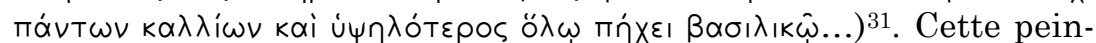
ture s'oppose à celle que lui réserve son séjour dans le monde inférieur. "Mais", - et ce "mais" est la marque de la satire "lorsqu'après sa mort je l'ai vu dépouillé de son faste, il ne m'a plus paru qu'un objet ridicule et je n'ai pu m'empêcher de rire de ma simplicité, qui me faisait admirer un scélérat, et juger de sa félicité par l'odeur de sa cuisine et par la couleur de ses habits teints du sang de coquillages qu'on trouve dans la mer de Laconie" (Lucien 2005).

Étant donné ce sens que Lucien attribue au surhomme, démasqué ou, pour utiliser le mot de Lucien, dépouillé de tout (statut et possessions, vêtements, apparence corporelle, muscles, chair et sang), le passage de la vie à la mort pousse la question de la démocratisation à son extrême limite. Les Grecs n'enseignaient pas la résurrection parfaite du corps (avoir un corps ressuscité après le trépas exige des convictions chrétiennes) mais l'ombre nue du moi comme étant déjà un accomplissement extraordinaire ${ }^{32}$ : le monde des Enfers est un monde d'ombres. Mais, par ce moyen, Lucien passe un excellent moment avec des crânes et des os : déclassant la beauté d'une Hélène, les prouesses guerrières d'un Achille, l'obscurité, comme le remarque Lucien, rend difficile la rencontre de ses voisins.

31. Lucien de Samosate (1913-67 : II, 34) Voir le « Ménippe ou le Voyage aux Enfers [ou La Nécyomancie]", dans le vol. IV. Cf. Nietzsche, $H H$, "Opinions et sentences mêlées", § 408, et Erwin Rohde (1900). Pour l'influence de Lucien, voir également Hunger (1978: 151 sq.), ainsi que Robinson (1979) en plus de Baumbach (2002) et, plus largement, von Koppenfels (2007). L'approche de Francis G. Allinson (1926) est enrichissante ; il se réfère, pour sa part, aux études de Rohde et à la dette très " lucianique » de Swift envers Lucien.

32. Comme le dit Marc-Aurèle, le problème avec la persistance des émanations du souffle ou de l'esprit est qu'elles prennent aussi de l'espace, et qu'elles se dissipent également. 
Pourtant, avec l'Übermensch, nous parlons de ce qui est probablement la plus influente des notions philosophiques nietzschéennes - largement plus influente que l'éternel retour du même, assez difficile à saisir. Insérée dans ce que certains prétendent avoir été son ultime constellation dans La volonté de puissance (ce livre notoirement "inventé »), l'idée même de l'Über-mensch dans son sens aryen peut être tenue pour responsable non seulement de la guerre d'Hitler, mais aussi de la Première Guerre mondiale (baptisée, c'est instructif, " la guerre de Nietzsche " par certains journalistes de l'époque $\left.{ }^{33}\right)$. Ce n'est pas non plus un hasard si George Bernard Shaw intitule l'une de ses pièces Homme et surhomme.

En parlant de l'Übermensch nietzschéen, on semble inévitablement parler de la philosophie qui a donné naissance au langage de la race des maîtres : l'Übermensch en tant qu'opposé à l'UnterMensch, comme le dit la terminologie Nationale Socialiste.

Nietzsche utilise les deux termes. La référence à Lucien suggère que le Zarathoustra de Nietzsche enseigne aussi l'Übermensch de manière parodique. Le surhumain est donc aussi une notion satirique, et cela revient à proposer une clarification qui en réalité clarifie bien peu le sens de Nietzsche. Ce que je défends ne rend pas les choses plus simples. Je ne suis pas en train de dire que Zarathoustra n'enseigne pas l'Übermensch, mais il est facile de ne pas voir que l'insaisissable doctrine de l'éternel retour, la doctrine que Zarathoustra vient pour enseigner, l'enseignement que le surhumain est destiné à permettre d'atteindre, est l'éternel retour du même. Ce point est également le cœur de la "vérité» de la renaissance chez Empédocle. Le Zarathoustra de Nietzsche enseigne que l'humain doit surmonter, c'est-à-dire aller au-delà, dépasser l'éternellement récurrent, et nous savons qu'il s'agit-là de l'humain, trop humain.

\section{Sur la mort : Zarathoustra, les îles Fortunées et la porte de l'Enfer}

Plus que l'avertissement d'Empédocle contre le carnivorisme, plus que son cycle cosmologique, c'est peut-être la description du volcan et de la mort volontaire du philosophe qui nous frappe le plus fortement. Il y a également le détail théâtral de son unique sandale de bronze, rejetée et rendue à la terre des vivants par le même volcan. Pourquoi n'a-t-elle pas été vaporisée ou fondue ? En effet, on attribue à Empédocle un pied de bronze, souvent évoqué en lien avec la cuisse d'or de Pythagore.

Le problème de la sandale subsiste. Pourquoi une seule? Les

33. Voir Salter (2009). Voir également l'ensemble de l'« Introduction " de Strong (xi-xxxiii) et ma propre analyse de Salter (Babich 2009). 
spécialistes suggèrent qu'il s'agit-là d'un signe ${ }^{34}$. Et le Diogène Laërte que Nietzsche fait sien évoque la sandale "rejetée " comme un signe grâce auquel, "plus tard ", la vérité pourra éclater. Ainsi, Jason $^{35}$ aussi perd une sandale en franchissant le fleuve boueux Anauros, alors qu'il porte sur l'autre rive une vieille femme qui s'avérera être Héra en personne (ce qui n'est pas un petit exploit, et il existe des mythes nordiques et chrétiens du même type, traitant de divinités déguisées comme les derniers des derniers et qui parviennent à se faire porter pour traverser divers flots).

De façon remarquable, en se souvenant de Lucien, la perte d'une sandale revêt un sens très particulier dans ce contexte. Ici, le passage concerne le Styx et le bronze de la sandale lancée en l'air signale un passage, un transfert vers les Enfers. Ainsi, le fait que les sandales d'Empédocle soient en métal - celles d'Hermès sont d'argent ou d'or, ce qui convient à un messager qui passe du royaume des morts à celui des vivants, mortels o immortels ${ }^{36}$ rattache le personnage au monde des Enfers. Souvenons-nous aussi qu'Hécate est justement associée à une unique sandale de bronze. Tout ceci a donc de l'importance par rapport à Lucien qui nous rappelle que Diogène le cynique (ici Lucien synthétise certains recensions) meurt pour avoir consommé (sous-entendu volontairement) le dîner d'Hécate - de la viande de chien - à un carrefour, évidemment. Si cet exposé était plus long, nous pourrions nous acheminer vers une réflexion sur le sens du hurlement du chien dans le Zarathoustra de Nietzsche, ainsi que sur les plaintes du "chevrier théocritien" dévoré par les punaises, dans les "chansons du prince hors-la-loi » en appendice au Gai savoir, tout comme sur la signification des lamentations des amants abandonnés. Je pense par exemple à Virgile faisant maudire Énée par Didon, avant sa propre mort volontaire, et qui invoque Hécate et quiconque aurait de la sympathie pour des amants délaissés : Didon que l'on voit « auprès des autels, un pied nu " (Énéide, IV) ${ }^{37}$.

Dans le deuxième livre d'Ainsi parlait Zarathoustra, le chapitre «Aux îles Fortunées " fait suite au début du renoncement de Zara-

34. Je suis reconnaissante à Bracht Branham de m'avoir indiqué cet aspect symbolique...

35. Je remercie plus particulièrement Peter Bing pour avoir mis en lumière l'allusion très importante, à mon sens, à Jason lorsque j'ai présenté une version de ce travail à la Emory University le 15 avril 2011.

36. Remarquons que les Grecs, comme les Hindous aujourd'hui, accordaient une valeur spirituelle au métal. Je propose de plus amples références dans Babich (2008 : 155-156).

37. Voir, sur la question de la sandale (peu discutée), Wolfgang Fauth (1985-86), ainsi que, pour un panorama général récent, Jacob Rabinowitz (2005). De son côté, Rabinowitz tire ses conclusions de, entre autres sources, Billault (1986) et Deborah Boedekker (1983). 
thoustra - une retraite qui dure des années - et s'achève par ces mots : " Du surhomme la beauté vint à moi comme une ombre ${ }^{38}$ ".

On connaît déjà le topos homérique des « îles Fortunées » par le sous-titre de l'Ardinghello de Wilhem Heinse, à qui Hölderlin dédie son Pain et vin et dont la même vision illumine le roman d'Hölderlin, Hypérion. Les contours géographiques de ces deux versions - Heinse exposant les souvenirs d'Ardinghello, un voyageur en Sicile, et ceux de l'Hypérion d'Hölderlin, l'ermite dans une vision idéalisée de la Grèce moderne - sont essentiels aux deux récits qui se réfèrent à la nostalgie d'un lieu et d'un monde transfigurés ${ }^{39}$. Hormis le "cadre " propre à la littérature allemande, on peut établir un parallèle avec l'œuvre du peintre suisse Arnold Böcklin (1827-1901), qui habita Bâle au même moment que Nietzsche $^{40}$. Bien que Böcklin ait également peint une version de L'̂̀le de vie, la Lebensinsel, sa toile la plus célèbre est la Toteninsel, L'île des morts, dont il réalisa plusieurs versions entre 1880 et 1886 . Ici la référence à Zarathoustra est liée à son propre vol dans le volcan (qui fait écho au suicide d'Empédocle), situé en un lieu précis de la Méditerranée. La plupart d'entre nous se souvient de ce passage de Zarathoustra en raison de sa bizarrerie ; plus étrangement encore, la majorité des spécialistes de Nietzsche (à l'exception de Jung [1988 : 1214]) ne font que rarement remarquer son extravagance. Je me souviens pour ma part de ma première lecture de ce passage, comme des centaines de lectures qui ont suivi, mais toujours sans lui trouver beaucoup de sens. Cela mérite qu'on s'y arrête, en particulier lorsqu'il s'agit de Nietzsche, qui a passé sa vie à s'occuper de bizarreries auxquelles la critique spécialisée a accordé peu d'attention ${ }^{41}$.

C'est dans ce contexte que l'on peut se tourner vers De grands événements, préfacé à dessein par la section Des poètes qui, sur un mode platonico-socratique, taquine les poètes pour leurs mensonges, tout en affirmant que "Zarathoustra lui-même est un poète " (Z II : Des poètes). Ainsi Zarathoustra met en garde son disciple contre la croyance, reflétant un mode que nous avons vu plus haut être "trop-lucianique», "mais suppose qu'en tout sérieux quel-

38. Notons que Zarathoustra se réfère plus loin à l'ombre et au monde de l'esprit comme à un choix du corps, en pensant : "à l'un de ses disciples, pour moi l'esprit n'est plus esprit que par manière de dire, et tout l'“impérissable" - cela aussi n'est qu'une image " (Z II : Des poètes).

39. Il existe de nombreuses études sur ce thème, à commencer par celle d'Eliza Butler (1958). Voir également, pour un exposé plus récent, Constanze Güthenke (2008 : 70 sq.).

40. Dans les notes de 1881, Nietzsche loue Böcklin comme " peintre explorant des voies nouvelles" (KSA 9, 11 [249], p. 536).

41. En règle générale, quand les spécialistes disent qu'ils sont "perplexes ", ils ne sont plus très loin d'écarter la question. 
qu'un dise que trop menteurs sont les poètes, celui-là n'a pas tort nous sommes trop menteurs" (ibid.). Comme nous le faisions remarquer en introduction, une telle réflexion sur le mensonge poétique est l'épithète de Lucien et le prétexte de l'Histoire véritable. Pour Zarathoustra (et ici pour Lucien) « trop pauvre est également notre savoir, et mal nous apprenons : ainsi déjà ne pouvons que mentir » (ibid.).

Associé à l'interprétation de Lucien et à la suggestion que Nietzsche réitère les purifications et la mort d'Empédocle avec Ainsi parlait Zarathoustra, le cadre initial de De grands événements commence par une étrangeté : c'est la constellation d'une île et d'un volcan du monde inférieur, d'un bateau, de son capitaine et son équipage, de la force de l'appel « Voici le temps ! Voici le temps suprême!" et, par dessus tout, de Zarathoustra volant dans les airs (passage accompagné du commentaire "c'est vers l'enfer que Zarathoustra chemine !»), mais aussi du laps de temps avant son retour le cinquième jour, alors que l'on avait fini par craindre que "que le diable [ait] enlevé Zarathoustra » ${ }^{42}$.

Rappelons-nous le début de la section : "Il est une île dans la mer - non loin des îles Fortunées de Zarathoustra - où ne cesse de fumer un volcan et dont le peuple dit, et singulièrement les vieilles petits femmes du peuple, que devant le portail du monde souterrain elle est placée comme un rocher, mais qu'à travers le volcan même descend l'étroit chemin qui mène à ce portail du monde souterrain » (Z II : De grands événements).

Ce passage est lié de manière évidente à Lucien, si nous sommes de surcroît guidés par le Psyche de Rohde (1925) ${ }^{43}$. De plus, il existe un conte de la même époque, conte de fantôme, qui renvoie à ces mêmes "grandes" (ou étranges) rencontres, comme on le découvre en poursuivant la lecture :

[...] il advint qu'un vaisseau jeta l'ancre près de l'île où se dresse la fumante montagne; et que fit terre son équipage pour tirer le lapin. Mais vers midi, lorsque le capitaine et ses hommes furent à nouveau réunis, en l'air ils virent soudain un homme qui s'approchait et une voix clairement dit "Voici le temps! Voici le temps suprême!". Or, comme cette forme était au plus près d'eux - elle volait cependant avec rapidité - ils reconnurent, très vivement émus, que c'était Zarathoustra (ibid.)

Ici l'histoire, telle que la raconte Nietzsche dans Ainsi parlait

42. La remarque est suivie de la réplique tout à fait ménippéenne (ou bien lucianique) "J'aime mieux croire que c'est Zarathoustra qui a enlevé le diable. "

43. L'étude de Rohde, originellement publiée au milieu des années 1890, est postérieure à ce que Nietzsche écrit ici, mais Nietzsche était familier du premier livre de Rohde sur Lucien et le roman grec et ses antécédents. 
Zarathoustra, reprend une légende populaire mettant en scène des fantômes, publiée par le poète souabe Justinus Kerner, qui luimême affirme, dans le Blätter aus Prevorst ${ }^{44}$, que cette histoire a d'abord été « reçue " par une voyante :

Les quatre capitaines et un marchand, Mr Bell, jetèrent l'ancre sur l'île de Stromboli pour tirer des lapins. À trois heures ils rappelèrent l'équipage pour monter à bord, quand, à leur plus inexprimable étonnement, ils virent deux hommes voler rapidement au-dessus d'eux dans les airs. L'un était habillé de noir, l'autre de gris. Ils les approchèrent très près, en grande hâte, et à leur plus grand désarroi ils descendirent dans les flammes brûlantes du cratère du terrible volcan, le Mont Stromboli. Ils reconnurent alors deux connaissances de Londres ${ }^{45}$.

Jung, qui affirme que "de telles histoires de fantômes perdurent parce qu'elles sont édifiantes", explique que "les deux gentilshommes de Londres étaient de célèbres commerçants, et de toute évidence ils n'allaient pas très bien, parce qu'ils étaient peints des couleurs de l'enfer qui expriment le péché, l'un en noir, l'autre en gris, alors qu'ils auraient dû porter des chemises blanches, qui sont les vêtements de cour au paradis" (ibid.) ${ }^{46}$.

Les Grüselgeschichten, contes de la mort, et particulièrement des morts malheureux, et des choses que nous ne savons pas en cette vie, comme c'est le cas à la fois du Kataplous de Lucien et du Psyche de Rohde, sont pareillement "instructifs» (ou édifiants). La dimension fantomatique du vol de Zarathoustra, comme la référen-

44. Bien que la citation soit donnée par le compilateur du Nietzsche's Zarathustra de Jung (1988: 2117) comme "The Seeress of Prevorst", la citation est tirée de Blätter aus Prevorst; Originalien und Lesefrüchte für Freunde des innern Lebens de Justinus Kerner, puisque ceux-ci constituent une série de volumes et que la référence de Jung est au « volume IV, p. 57 ». Je remercie Robin Small pour avoir souligné ce détail.

45. Jung (1988 : 1217-1218). Remarquant que cette histoire est la même que chez Nietzsche, Jung vérifie auprès d'Elisabeth Förster-Nietzsche, qui confirme qu'elle et son frère ont lu ce livre dans la bibliothèque de leur grand-père, le pasteur Oehler. Jung demanda en premier lieu à FörsterNietzsche parce qu'il avait reconnu l'histoire, à partir de la même source, dans la bibliothèque de son propre grand-père (ibid. : 2118). Étant donné le fétichisme qui semble entourer la recherche des sources de Nietzsche (que ce soit pour prouver ou mettre en doute son originalité), on pouvait s'attendre à ce que les commentateurs soient prompts à se demander si Elisabeth mentait, mais mentir n'aurait ici servi aucun objectif. En fait, la coïncidence est plausible dans la confirmation d'Elisabeth, et BennholdtThomsen remarque aussi que Nietzsche s'intéressa à Kerner entre l'âge de douze et quinze ans.

46. Je remercie Annette Hornbacher pour avoir relevé cette référence de Jung sur la différence des couleurs, dont la signification est elle-même tirée de Kerner. 
ce ci-dessus à Lucien et à Rohde le montre bien, est plus littérale que littéraire. Si Gary Shapiro, un des rares chercheurs à écrire à ce sujet, souligne très justement la signification géologique de ce passage face à celui des îles Fortunées, et précise qu'ici, dans De grands événements, "Zarathoustra apparaît mystérieusement sur une île volcanique (où son Ombre semble voler dans le volcan luimême) " (2008:13), il ne remarque pas que l'ombre de Zarathoustra, l'Ombre en question, correspond à la dimension aplatie de ce qui est pour le Grec de l'antiquité la seule chose qui subsiste de nous après la mort.

Ainsi, le "temps suprême," invoqué par le Zarathoustra de Nietzsche - "C'est l'heure, c'est l'heure " [it's time, it's time] comme le disait T. S. Eliot - désigne le moment de la mort ${ }^{47}$. C'est dans notre " solitude la plus solitaire" (sachant que chacun doit mourir sa propre mort, ce qui signifie, comme le dira Heidegger, que personne ne peut mourir pour vous : la mort est à chaque fois seulement mienne ${ }^{48}$ ) qu'il faut entendre l'enseignement de Zarathoustra sur l'éternel retour du même. Et de cet enseignement on peut dire, comme Nietzsche le fait: que vous l'entendiez ou non, c'est ce que vous ferez de toute façon - Du wirst es jedenfalls.

\section{Coda}

Ce que Nietzsche retient du Kataplous de Lucien est l'opposition entre les valeurs de notre culture et notre tendance à prendre ces valeurs, dans la manière du présentisme que décrit Herbert Butterfield, non seulement comme universelles, mais aussi comme éternelles. En écrivant que l'homme doit être dépassé, Nietzsche tend vers un au-delà de l'ici et maintenant, il nous demande de prendre en compte non pas l'immédiat, qu'il soit fait d'avantages économiques ou de plaisirs, mais le cosmos comme volonté de puissance.

Nietzsche ne dit pas "Le petit homme d'affaires est volonté de puissance et rien d'autre", encore moins "Le nationaliste allemand est volonté de puissance et rien d'autre", mais "Le monde est volonté de puissance et rien d'autre " - signifiant par là la totalité collective de l'existence dans le monde (et en réalité au-delà de la terre). Il insiste sur le fait que le but de la vie est l'expression et non la survie. Il n'y a que les Anglais, ajoute-t-il un peu méchamment, qui recherchent la vie coûte que coûte. Mais il s'agit-là d'une

47. Jung (1988: 1224) explique que « C'est le secret, c'est la clef de la signification de cette descente aux enfers. C'était un avertissement : bientôt vous serez dissouts."

48. «Tod ist je nur eigner », Heidegger (1984: 265). Günther Anders fait néanmoins remarquer que cela s'applique à un certain nombre d'autres choses, comme faire l'amour, manger des gâteaux, etc. 
critique générale de l'ascétisme en tant qu'opposé à la vie. "Vivre", comme Nietzsche le note, "veut dire : rejeter sans cesse loin de soi quelque chose qui veut mourir ... être sans cesse un assassin " (GS §26, traduction modifiée), mais comprend aussi et toujours la mort elle-même. La vie est faite de devenir, de changement, croissance, reproduction, et de mort, comme Nietzsche les énumère dans le Crépuscule des idoles en les opposant à l'idée fixe de " la "raison" en philosophie».

On peut se demander ce que Nietzsche a pu vouloir dire en faisant de Zarathoustra un sage pour un monde moderne, et même de façon plus radicale en n'appelant ni au salut ni à un remède mais, et en premier lieu, à une purification. Or pour comprendre cet appel, nous devons dépasser l'humain trop humain, et pour cela nous avons besoin d'apprendre à aimer la vie au-delà de l'humanité.

Babette BABICH. (Fordham University.)

(Traduit de l'anglais par Brigitte Rollet \& Anne-Gaëlle Argy, en collaboration avec Tracy B. Strong et l'auteur.)

\section{Références}

Allinson, Francis G. (1926) Lucian: Satirist and Artist. Boston : Marshall Jones Company.

Babich, B. (2005) "The Science of Words or Philology: Music in The Birth of Tragedy and The Alchemy of Love in The Gay Science ", Rivista di estetica, $28: 47-78$.

Babich, B. (2008) « Die Naturkunde der Griechischen Bronze im Spiegel des Lebens: Betrachtungen über Heideggers ästhetische Phänomenologie und Nietzsches agonale Politik", trad. H. Seubert avec l'auteur, dans: Günter Figal (éd.) Internationales Jahrbuch für Hermeneutik, p. 127-189. Tübingen : Mohr Siebeck.

Babich, B. (2009) "Nietzsche's Will to Power: Politics and Destiny", dans : Tracy Strong (éd.) Friedrich Nietzsche, p. 281-296. Aldershot: Ashgate.

Babich, B. (2010) « Towards a Critical Philosophy of Science: Continental Beginnings and Bugbears, Whigs and Waterbears", International Journal of the Philosophy of Science, 24(4) : 343-391.

Baier, Annette (2008) Death and Character: Further Reflections on Hume. Cambridge, MA : Harvard UP.

Bartley, Adam, éd. (1989) A Lucian for Our Times. Newcastle-uponTyne : Cambridge Scholars.

Baumbach, Manuel (2002) Lukian in Deutschland. Eine forschungsund rezeptionsgeschichtliche Analyse vom Humanismus bis zur Gegenwart. Munich : Wilhelm Fink .

Billault, A. (1986) "Hécate Romanesque", dans : Mort et Fécondité dans les Mythologies. Actes du Colloque de Poitiers, 13-14 mai 1983, p. 109-16. Paris : Les Belles Lettres. 
Boedekker, Deborah (1983) « Hecate: A Transfunctional Goddess in the Theogony? » TAPA, $113:$ 79-93.

Branham, Robert Bracht (1989) Unruly Eloquence: Lucian and the Comedy of Traditions. Cambridge : Cambridge UP.

Butler, Eliza M. (1958) Tyranny of Greece over Germany. Boston : Beacon press.

Butterfield, Herbert (1951) The Whig Interpretation of History. Londres : Bell.

Cardew, Alan (2004) "The Dioscuri: Nietzsche and Erwin Rohde", dans : Paul Bishop (éd.), Nietzsche and Antiquity, p. 458-478. Rochester : Boydell \& Brewer.

Clay, Diskin (1992) «Lucian of Samosata: Four Philosophical Lives (Nigrinus, Perigrinus, Demonax, Alexander Pseudomantis) », Aufstieg und Niedergang der römischen Welt, 2.36 .5 : 3406-3450.

Derrida, Jacques (1989) "Il faut bien manger" ou le calcul du sujet. entretien avec Jean-Luc Nancy ", Cahiers Confrontation 20 : 91-114.

Diogène Laërte (1965) Vie, doctrines et sentences des philosophes illustres, trad. R. Grenaille. Paris : Garnier.

Ebner, Martin, Gzella, Holger, Nesselrath, Heinz-Günther et Ribbat, Ernst, éds (2001) Philopseudeis è Apiston. Die Lügenfreunde oder: Der Ungläubige. Darmstadt, Wissenschaftliche Buchgesellschaft.

Eisler, Rudolf (1904) Handwörterbuch der Philosophie. Berlin : Mittler und Sohn.

Empédocle (1934) Katharmoi, dans : Hermann Diels \& Walther Kranz, Die Fragmente der Vorsokratiker, Vol. 1, p. 354 s. Berlin : Wiedmannsche Buchhandlung.

Fauth, Wolfgang (1985-86) «Aphrodites Pantoffel und die Sandale der Hekate " Grazer Beiträge, 12-13 : 193-211.

Frye, Northrop (1969) Anatomie de la critique. Paris : Gallimard.

Ginzburg, Carlo (2005) Nulle île n'est une île : quatre regards sur la littérature anglaise. Lagrasse : Verdier.

Güthenke, Constanze (2008) Placing Modern Greece: The Dynamics of Romantic Hellenism, 1770-1840. Oxford : Oxford UP.

Habermas, Jürgen (2001) Die Zukunft der menschlichen Natur. Auf dem Weg zu einer liberalen Eugenik? Francfort sur Main : Suhrkamp.

Heidegger, Martin (1984) Sein und Zeit. Tübingen : Niemeyer.

Hock, Ronald F. (1987) "Lazarus and Micyllus: Greco-Roman Backgrounds to Luke 16 : 19-31 », J. of Biblical Literature, 106(3) : 447-463.

Hunger, Herbert (1978) Die hochsprachliche profane Literatur der Byzantiner; Teilbd. 2 : Philologie, Profandichtung, Musik, Mathematik und Astronomie, Naturwissenschaften, Medizin, Kriegswissenschaft, Rechtsliteratur. Munich : Beck.

Jung, Carl Gustav (1988) Nietzsche's Zarathustra: Notes of the Seminar Given in 1934-1939, trad. James L. Jarrett. Princeton, NJ : Princeton UP.

Kainz, Friedrich (1974) "Klassik und Romantik», dans : Friedrich Maurer et Heinz Rupp (éds) Deutsche Wortgeschichte, t. 2, p. 245-491. Berlin : de Gruyter.

Kaufmann, Walter A. (1974) Nietzsche, Philosopher, Psychologist, Antichrist. Princeton, NJ : Princeton UP.

von Koppenfels, Werner (2007) Der andere Blick. Das Vermächtnis des Menippos in der europäischen Literatur. Munich : Beck. 
Lauvergnat-Gagnière, Christine (1988) Lucien de Samosate et le Lucianisme en France au XVI siècle : Athéisme et polémique. Genève : Droz.

Lucien de Samosate (1866) Euvres Complètes. 2e éd. Paris : Hachette ; http://remacle.org/bloodwolf/philosophes/Lucien/veritable1.htm.

Lucien de Samosate (1896) Euvres Complètes, traduction de Belin de Ballu, t. 1. Paris : Garnier.

Lucien de Samosate (1913-67) Lucian, with an English translation by A. M. Harmon, 8.v. Cambridge, MA : Harvard University Press.

Lucien de Samosate (1968) Selected Satires of Lucian, trad. Lionel Casson. New York : Norton.

Lucien de Samosate (2005) La Traversée pour les Enfers, nouv. traduction de Philippe Renault, http://bcs.fltr.ucl.ac.be/LUCIEN/Traversee.html.

Nietzsche, F. (1971) Euvres philosophiques complètes, édition sous la direction de Gilles Deleuze et Maurice de Gandillac. Paris : Gallimard.

Nietzsche, F. (1980) Kritische Studien Ausgabe [KSA]. Berlin: De Gruyter.

Pavur, Claude (1998) Nietzsche Humanist. Milwaukee : Marquette UP.

Rabinowitz, Jacob (2005) "The "Her" Story of the Great WitchGoddess: Analyzing the Narratives of Hekate", AJCN, 2, http://cf.hum.uva.nl/narratology/a05_rabinowitz_00.html.

Rauer, Konstantin (2005) « Totengespräch zwischen Kant and Nietzsche zur Moralphilosophie", dans : Beatrix Himmelmann (éd.), Kant und Nietzsche im Widerstreit, p. 119-129. Berlin : de Gruyter.

Renault, Philippe (2004) « Lucien de Samosate ou le prince du gai savoir » Folia Electronica Classica, 8, bcs.fltr.ucl.ac.be/FE/08/Lucien.html.

Robinson, Christopher (1979) Lucian and his Influence in Europe. Londres : Duckworth.

Rohde, Erwin (1869) Über Lucians Schrift Loukios e Onos und ihr Verhältnis zu Lucius von Patrae und den Metamorphosen des Apuleius. Leipzig : Engelmann.

Rohde, Erwin (1900) Der griechische Roman und seine Vorläufer. Leipzig : Breitkopf und Härtel.

Rohde, E. (1925) Psyche, Seelencult und Unsterblichkeitsglaube der Griechen. Tübingen : Mohr.

Salter, William MacIntire (2009) "Nietzsche and War», dans : Tracy Strong (éd.) Friedrich Nietzsche, p. 3-26. Aldershot : Ashgate.

Shapiro, Gary (2008) "Beyond Peoples and Fatherlands: Nietzsche's Geophilosophy and the Direction of the Earth ", Journal of Nietzsche Studies, 35-36 : 9-27.

Wallas, Armin A. (1994) Albert Ehrenstein: Mythenzerstörer und Mythenschöpfer. Grafrath : Boer.

Wienand, Isabelle (2006) Significations de la Mort de Dieu chez Nietzsche $d$ 'Humain, trop humain à Ainsi parlait Zarathoustra. Bern/Oxford : Peter Lang.

Wolfe, Peter (1964) "Image and Meaning in Also Sprach Zarathustra », MLN, 89(5) : 546-552. 